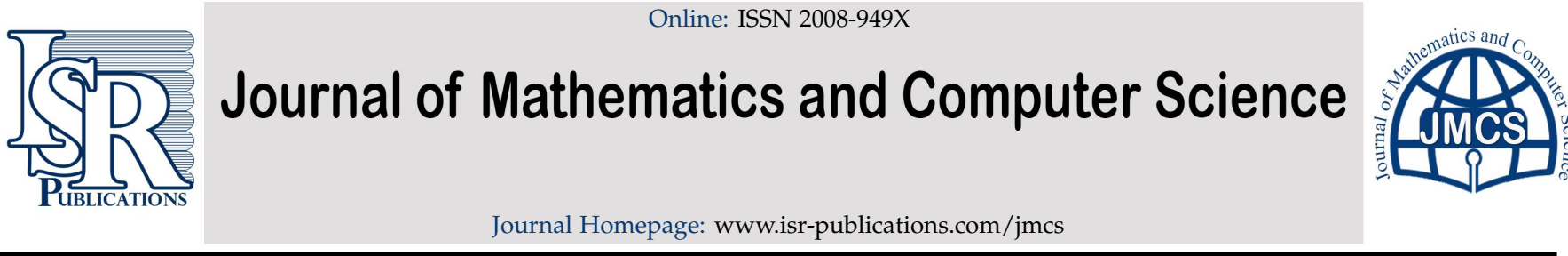

\title{
Note on the stability property of the boundary equilibrium of a May cooperative system with strong and weak coopera- tive partners
}

\author{
Runxin $\mathrm{Wu}^{*}$, Lin Li
}

School of Mathematics and Physics, Fujian University of Technology, Fuzhou, Fujian 350014, China.

\begin{abstract}
In this paper, we revisit the stability property of the boundary equilibrium of a May cooperative system with strong and weak cooperative partners. Our result essentially improves the corresponding result of Zhao et al. [L. Zhao, B. Qin, F. D. Chen, Adv. Difference Equ., 2018 (2018), 13 pages].
\end{abstract}

Keywords: Cooperative system, boundary equilibrium, global stability.

2010 MSC: 34C25, 92D25, 34D20, 34D40.

(C)2020 All rights reserved.

\section{Introduction}

During the last decade, many scholars ([2-14, 16-25]) investigated the dynamic behaviors of the mutualism and commensalism model. Some substantial progress has been made on the stability, permanence and extinction of the mutualism model. For example, under some very simple assumptions, Xie et al. [16] showed that unique positive equilibrium of a cooperative system incorporating harvesting is globally attractive; Xie et al. [17] showed that the unique positive equilibrium of an integrodifferential model of mutualism is globally attractive. Recently, stimulated by the idea of Mohammadi and Mahzoon [15], Zhao et al. [25] proposed the following May cooperative system with strong and weak cooperative partners

$$
\begin{aligned}
\frac{d H_{1}}{d t} & =r_{1} H_{1}\left(1-\frac{H_{1}}{a_{1}+b_{1} P}-c_{1} H_{1}-\frac{\alpha H_{2}}{r_{1}}\right), \\
\frac{d H_{2}}{d t} & =H_{2}\left(\alpha H_{1}+d-e H_{2}\right), \\
\frac{d P}{d t} & =r_{2} P\left(1-\frac{P}{a_{2}+b_{2} H_{1}}-c_{2} P\right),
\end{aligned}
$$

\footnotetext{
*Corresponding author

Email address: 18106069131@163.com (Runxin Wu)
}

doi: $10.22436 /$ jmcs.020.01.07 
where $r_{i}, a_{i}, b_{i}, c_{i}, d, i=1,2$ are positive constants. We will consider system (1.1) together with the initial condition $\mathrm{H}_{i}(0)>0, i=1,2, \mathrm{P}(0)>0$ in system (1.1). Obviously, any solution of system (1.1) remains positive for all $t \geqslant 0$. One could refer to [25] for more detail about the construction of the model.

The system (1.1) always admits a boundary equilibrium $\mathrm{E}_{2}\left(0, \mathrm{H}_{2 *}, \mathrm{P}_{*}\right)$ (see Theorem 3.2 in [25]), indeed, by simple computation, one could see that $\mathrm{H}_{2 *}=\frac{\mathrm{d}}{\mathrm{e}}, \mathrm{P}_{*}=\frac{\mathrm{a}_{2}}{1+\mathrm{a}_{2} \mathrm{c}_{2}}$. Concerned with the stability property of this equilibrium, by constructing some suitable Lyapunv function, the authors obtained the following results (see Theorem 4.2 in [25] for more details).

Theorem 1.1. If the assumption $\left(\mathrm{B}_{3}\right)$ and $\left(\mathrm{B}_{5}\right)$ hold, where

$\left(\mathrm{B}_{3}\right) \mathrm{M}=1-\frac{\alpha \mathrm{d}}{\mathrm{r}_{1} e}<0$;

$\left(B_{5}\right) \alpha^{2}<r_{1} c_{1} e$,

then the equilibrium point $\mathrm{E}_{2}\left(0, \mathrm{H}_{2 *}, \mathrm{P}_{*}\right)$ system is globally asymptotically stable.

Now let's consider the following example.

\section{Example 1.2.}

$$
\begin{aligned}
\frac{\mathrm{d} \mathrm{H}_{1}}{\mathrm{dt}} & =3 \mathrm{H}_{1}\left(1-\frac{\mathrm{H}_{1}}{2+2 \mathrm{P}}-\mathrm{H}_{1}-\frac{3.5 \mathrm{H}_{2}}{3}\right), \\
\frac{\mathrm{d} \mathrm{H}_{2}}{\mathrm{dt}} & =\mathrm{H}_{2}\left(3.5 \mathrm{H}_{1}+2-2 \mathrm{H}_{2}\right), \\
\frac{\mathrm{dP}}{\mathrm{dt}} & =2 \mathrm{P}\left(1-\frac{\mathrm{P}}{2+0.8 \mathrm{H}_{1}}-1.5 \mathrm{P}\right) .
\end{aligned}
$$

Here, corresponding to system (1.1), we take $r_{1}=3, a_{1}=2, b_{1}=2, c_{1}=1, \alpha=3.5, r_{1}=3, d=2, e=$ $2, r_{2}=2, a_{2}=2, b_{2}=0.8, c_{2}=1.5$. By simple computation, one could easily see that

$$
M=1-\frac{\alpha d}{r_{1} e}=-\frac{0.5}{3}=-\frac{1}{6}<0 \quad \text { and } \quad \alpha^{2}=12.25>6=r_{1} c_{1} e .
$$

That is, the coefficients of the system (1.2) satisfy the condition $\left(B_{3}\right)$, however, they do not satisfy the condition $\left(B_{5}\right)$, however, numeric simulations (Figs 1-3) show that in this case, the boundary equilibrium $\left(0,1, \frac{2}{3}\right)$ is globally attractive.

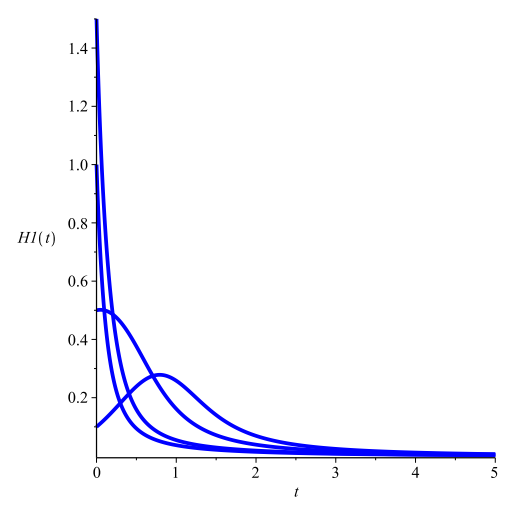

Figure 1: Numeric simulations of the first component system (1.2), the initial conditions $(x(0), y(0))=$ $(1,2,0.7),(1.5,1,0.3),(0.5,0.2,0.1)$ and $(0.1,0.1,2)$, respectively.

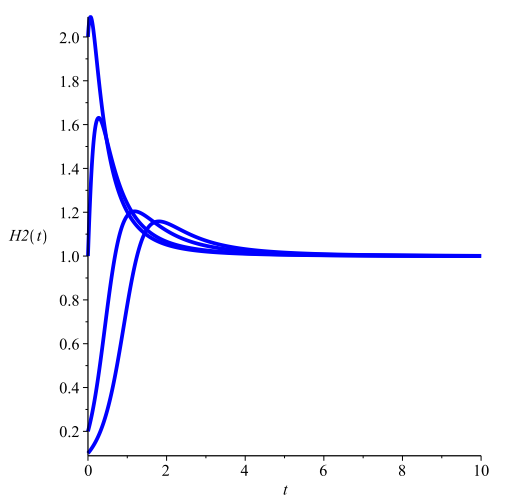

Figure 2: Numeric simulations of the second component system (1.2), the initial conditions $(x(0), y(0))=$ $(1,2,0.7),(1.5,1,0.3),(0.5,0.2,0.1)$ and $(0.1,0.1,2)$, respectively. 


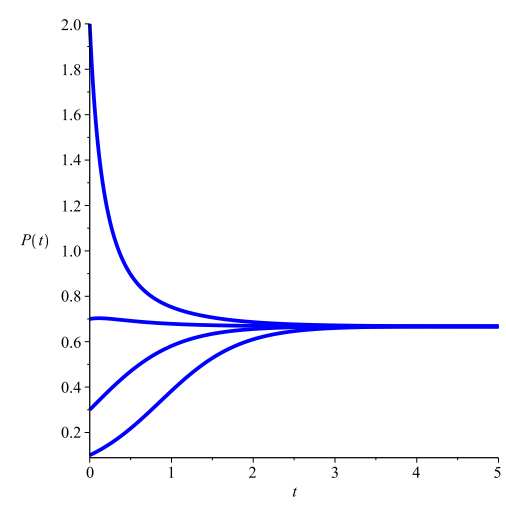

Figure 3: Numeric simulations of the third component system (1.2), the initial conditions $(x(0), y(0))=(1,2,0.7),(1.5,1,0.3),(0.5,0.2,0.1)$ and $(0.1,0.1,2)$, respectively.

Above numeric simulations show that there still have room to improve the main result ([25, Theorem 4.2]). The aim of this paper is try to obtain a new set of sufficient conditions to ensure the global attractivity of the positive equilibrium, more precisely, we will obtain the following result.

Theorem 1.3. If the assumption $\left(\mathrm{B}_{3}\right)$ holds, where

$\left(\mathrm{B}_{3}\right) \mathrm{M}=1-\frac{\alpha \mathrm{d}}{\mathrm{r}_{1} \mathrm{e}}<0$,

then the equilibrium point $\mathrm{E}_{2}\left(0, \mathrm{H}_{2 *}, \mathrm{P}_{*}\right)$ system is globally attractive.

Remark 1.4. Compared with Theorem 1.1 and Theorem 1.3, One could easily see that condition $\left(\mathrm{B}_{5}\right)$ is redundantly and unnecessary.

We will prove Theorem 1.3 in the next section and end this paper by a briefly discussion.

\section{Proof of the main result}

We need the following lemma to prove the main result.

Lemma 2.1 ([1]). Let $a>0, b>0$.

(I) If $\frac{d x}{d t} \geqslant x(b-a x)$, then $\liminf _{t \rightarrow+\infty} x(t) \geqslant \frac{b}{a}$ for $t \geqslant 0$ and $x(0)>0$;

(II) If $\frac{\mathrm{d} x}{\mathrm{~d} t} \leqslant x(b-a x)$, then $\limsup _{\mathrm{t} \rightarrow+\infty} x(t) \leqslant \frac{\mathrm{b}}{\mathrm{a}}$ for $\mathrm{t} \geqslant 0$ and $x(0)>0$.

Now we are in the position of proving the Theorem 1.3, we mention here that Zhao et al. [25] had already proved part of the results of Theorem 1.3, however, for the sake of completeness, here we give the detail proof of the Theorem 1.3.

Proof of Theorem 1.3. From the second equation of system (1.1), one has

$$
\frac{d H_{2}}{d t} \geqslant H_{2}\left(d-e H_{2}\right)
$$

applying Lemma 2.1 to (2.1) leads to

$$
\liminf _{t \rightarrow+\infty} H_{2}(t) \geqslant \frac{d}{e}
$$


Condition $\left(B_{3}\right)$ implies that there exists a enough small $\varepsilon>0$ such that

$$
1-\frac{\alpha}{r_{1}}\left(\frac{d}{e}-\varepsilon\right)<0
$$

Indeed, for all $\varepsilon$ which satisfies $0<\varepsilon<\frac{\frac{\alpha d}{r_{1} e}-1}{\frac{\alpha}{r_{1}}}$, inequality (2.3) holds. For this $\varepsilon$, from (2.2) there exists an enough large $T_{1}$ such that

$$
\mathrm{H}_{2}(\mathrm{t})>\frac{\mathrm{d}}{\mathrm{e}}-\varepsilon \text { for all } \mathrm{t} \geqslant \mathrm{T}_{1} .
$$

From (2.4) and the first equation of system (1.1), for $t>T_{1}$, one has

$$
\frac{d H_{1}}{d t}<r_{1} H_{1}\left(1-\frac{H_{1}}{a_{1}+b_{1} P}-c_{1} H_{1}-\frac{\alpha}{r_{1}}\left(\frac{d}{e}-\varepsilon\right)\right)<r_{1} H_{1}\left(1-\frac{\alpha}{r_{1}}\left(\frac{d}{e}-\varepsilon\right)\right) \text {. }
$$

Thus

$$
H_{1}(t)<H_{1}\left(T_{1}\right) \exp \left\{r_{1}\left(1-\frac{\alpha}{r_{1}}\left(\frac{d}{e}-\varepsilon\right)\right)\left(t-T_{1}\right)\right\} .
$$

It then follows from (2.3) that

$$
\lim _{t \rightarrow+\infty} H_{1}(t)=0 .
$$

For any $\varepsilon_{1}>0$ enough small, from (2.5), there exists an enough large $T_{2}>T_{1}$ such that

$$
H_{1}(t)<\varepsilon_{1} \text { for all } t>T_{2} .
$$

For $t>T_{2}$, from the second equation of system (1.1) and (2.6), it follows that

$$
\frac{d H_{2}}{d t}<H_{2}\left(\alpha \varepsilon_{1}+d-e H_{2}\right) .
$$

By applying Lemma 2.1 to (2.7), it immediately follows that

$$
\limsup _{t \rightarrow+\infty} H_{2}(t)<\frac{\alpha \varepsilon_{1}+d}{e} .
$$

Since $\varepsilon_{1}$ is any enough small positive constant, setting $\varepsilon_{1} \rightarrow 0$ in (2.8) leads to

$$
\limsup _{t \rightarrow+\infty} H_{2}(t) \leqslant \frac{d}{e} \text {. }
$$

(2.2) together with (2.9) shows that

$$
\frac{d}{e} \leqslant \liminf _{t \rightarrow+\infty} H_{2}(t) \leqslant \limsup _{t \rightarrow+\infty} H_{2}(t) \leqslant \frac{d}{e} .
$$

Hence

$$
\lim _{t \rightarrow+\infty} H_{2}(t)=\frac{d}{e} .
$$


For $t>T_{2}$, from the third equation of system (1.1) and (2.6), it follows that

$$
\frac{d P}{d t}<r_{2} P\left(1-\frac{P}{a_{2}+b_{2} \varepsilon_{1}}-c_{2} P\right) .
$$

By applying Lemma 2.1 to (2.11), it immediately follows that

$$
\limsup _{t \rightarrow+\infty} P(t)<\frac{1}{\frac{1}{a_{2}+b_{2} \varepsilon_{1}}+c_{2}} .
$$

Since $\varepsilon_{1}$ is any enough small positive constant, setting $\varepsilon_{1} \rightarrow 0$ in (2.12) leads to

$$
\limsup _{t \rightarrow+\infty} P(t) \leqslant \frac{1}{\frac{1}{a_{2}}+c_{2}}=\frac{a_{2}}{1+a_{2} c_{2}} .
$$

On the other hand, from the third equation of system (1.1), we also have

$$
\frac{d P}{d t}>r_{2} P\left(1-\frac{P}{a_{2}}-c_{2} P\right) .
$$

By applying Lemma 2.1 to (2.14), it immediately follows that

$$
\liminf _{t \rightarrow+\infty} P(t)>\frac{1}{\frac{1}{a_{2}}+c_{2}}=\frac{a_{2}}{1+a_{2} c_{2}} .
$$

(2.13) together with (2.15) shows that

$$
\frac{a_{2}}{1+a_{2} c_{2}} \leqslant \liminf _{t \rightarrow+\infty} P(t) \leqslant \limsup _{t \rightarrow+\infty} P(t) \leqslant \frac{a_{2}}{1+a_{2} c_{2}} .
$$

Hence

$$
\lim _{t \rightarrow+\infty} P(t)=\frac{a_{2}}{1+a_{2} c_{2}} .
$$

(2.5), (2.10), and (2.16) show that the equilibrium point $E_{2}\left(0, H_{2 *}, P_{*}\right)$ is globally attractive. This ends the proof of Theorem 1.3.

\section{Discussion}

In [25], Zhao et al. proposed the system (1.1), by constructing some suitable Lyapunov function, they obtained a set of sufficient conditions which ensure the global asymptotically stable of the boundary equilibrium $\mathrm{E}_{2}\left(0, \mathrm{H}_{2 *}, \mathrm{P}_{*}\right)$, by carefully checking the conditions, we found that $\left(\mathrm{B}_{3}\right)$ requires $\alpha$ enough large, that is, the translate rate of strong partners to weak partners is enough large, however, condition $\left(B_{5}\right)$ requires $\alpha$ enough small, that is, conditions $\left(B_{3}\right)$ and $\left(B_{5}\right)$ seem to contradict to each other, this motivated us to revisit the globally attractivity of the boundary equilibrium $E_{2}$, with the aim of finding some set of conditions which may seems no contract. By applying the theory of differential inequality, we finally show that condition $\left(B_{5}\right)$ is not needed and could be dropped. Our result (Theorem 1.3) essentially improve one of the main results of [25] (Theorem 4.2).

\section{Acknowledgment}

The research was supported by the Natural Science Foundation of Fujian Province (2019J01783). The authors would like to thank Dr. Yu Liu for useful discussion about the mathematical modeling. 


\section{References}

[1] F. D. Chen, On a nonlinear nonautonomous predator-prey model with diffusion and distributed delay, J. Comput. Appl. Math., 180 (2005), 33-49. 2.1

[2] B. G. Chen, Dynamic behaviors of a commensal symbiosis model involving Allee effect and one party can not survive independently, Adv. Difference Equ., 2018 (2018), 12 pages. 1

[3] B. G. Chen, The influence of commensalism on a Lotka-Volterra commensal symbiosis model with Michaelis-Menten type harvesting, Adv. Difference Equ., 2019 (2019), 14 pages.

[4] F. D. Chen, H. L. Wu, X. D. Xie, Global attractivity of a discrete cooperative system incorporating harvesting, Adv. Difference Equ., 2016 (2016), 12 pages.

[5] L. J. Chen, X. D. Xie, Permanence of an N-species cooperation system with continuous time delays and feedback controls, Nonlinear Anal. Real World Appl., 12 (2001), 34-38.

[6] F. D. Chen, X. D. Xie, X. F. Chen, Dynamic behaviors of a stage structured cooperation model, Commun. Math. Biol. Neurosci., 2015 (2015), 19 pages.

[7] F. D. Chen, Y. L. Xue, Q. F. Lin, X. D. Xie, Dynamic behaviors of a Lotka Volterra commensal symbiosis model with density dependent birth rate, Adv. Difference Equ., 2018 (2018), 14 pages.

[8] R. Y. Han, X. D. Xie, F. D. Chen, Permanence and global attractivity of a discrete pollination mutualism in plant pollinator system with feedback controls, Adv. Difference Equ., 2016 (2016), 17 pages.

[9] C. Q. Lei, Dynamic behaviors of a stage structured commensalism system, Adv. Difference Equ., 2018 (2018), 20 pages.

[10] X. P. Li, W. S. Yang, Permanence of a discrete model of mutualism with infinite deviating arguments, Discrete Dyn. Nat. Soc., 2010 (2010), 7 pages.

[11] Y. K. Li, T. W. Zhang, Permanence of a discrete n-species cooperation system with time-varying delays and feedback controls, Math. Comput. Modelling, 53 (2011), 1320-1330.

[12] Q. F. Lin, Allee effect increasing the final density of the species subject to the Allee effect in a Lotka Volterra commensal symbiosis model, Adv. Difference Equ., 2018 (2018), 9 pages.

[13] Z. J. Liu, J. H. Wu, R. H. Tan, Y. P. Chen, Modeling and analysis of a periodic delayed two species model of facultative mutualism, Appl. Math. Comput., 217 (2010), 893-903.

[14] Y. Liu, X. D. Xie, Q. F. Lin, Permanence, partial survival, extinction, and global attractivity of a nonautonomous harvesting Lotka Volterra commensalism model incorporating partial closure for the populations, Adv. Difference Equ., 2018 (2018), 16 pages. 1

[15] H. Mohammadi, M. Mahzoon, Effect of weak prey in Lesile-Gower predator-prey model, Appl. Math. Comput., 224 (2013), 196-204. 1

[16] X. D. Xie, F. D. Chen, Y. L. Xue, Note on the stability property of a cooperative system incorporating harvesting, Discrete Dyn. Nat. Soc., 2014 (2014), 5 pages. 1

[17] X. D. Xie, F. D. Chen, K. Yang, Y. L. Xue, Global attractivity of an integrodifferential model of mutualism, Abstr. Appl. Anal., 2014 (2014), 6 pages. 1

[18] X. D. Xie, Y. L. Xue, R. X. Wu, Global attractivity in a discrete mutualism model with infinite deviating arguments, Discrete Dyn. Nat. Soc., 2017 (2017), 8 pages.

[19] Y. L. Xue, X. D. Xie, F. D. Chen, R. Y. Han, Almost periodic solution of a discrete commensalism system, Discrete Dyn. Nat. Soc., 2015 (2015), 11 pages.

[20] W. S. Yang, X. P. Li, Permanence of a discrete nonlinear N-species cooperation system with time delays and feedback controls, Appl. Math. Comput., 218 (2011), 3581-3586.

[21] K. Yang, Z. S. Miao, F. D. Chen, X. D. Xie, Influence of single feedback control variable on an autonomous Holling II type cooperative system, J. Math. Anal. Appl., 435 (2016), 874-888.

[22] K. Yang, X. D. Xie, F. D. Chen, Global stability of a discrete mutualism model, Abstr. Appl. Anal., 2014 (2014), 7 pages.

[23] L. Y. Yang, X. D. Xie, F. D. Chen, Dynamic behaviors of a discrete periodic predator prey mutualist system, Discrete Dyn. Nat. Soc., 2015 (2015), 11 pages.

[24] L. Y. Yang, X. D. Xie, F. D. Chen, Y. L. Xue, Permanence of the periodic predator prey mutualist system, Adv. Difference Equ., 2015 (2015), 15 pages.

[25] L. Zhao, B. Qin, F. D. Chen, Permanence and global stability of a May cooperative system with strong and weak cooperative partners, Adv. Difference Equ., 2018 (2018), 13 pages. 1, 1, 1, 2, 3 